\title{
The epsilon-reconstruction of theories and scientific structuralism
}

August 1, 2015

\begin{abstract}
Rudolf Carnap's mature work on the logical reconstruction of scientific theories consists of two components. The first is the elimination of the theoretical vocabulary of a theory in terms of its Ramsification. The second is the reintroduction of the theoretical terms through explicit definitions in a language containing an epsilon operator. This paper investigates Carnap's epsilon-reconstruction of theories in the context of pure mathematics. The main objective here is twofold: first, to specify the epsilon logic underlying his suggested definition of theoretical terms and a suitable choice semantics for it. Second, to analyze whether Carnap's approach is compatible with a structuralist conception of mathematics.
\end{abstract}

\section{Introduction}

In the logical analysis of science, theories are often described as axiomatic systems formulated in a language whose vocabulary is bipartitioned into observational and theoretical terms. ${ }^{1}$ A central interpretive issue in the relevant literature since Carnap concerns the proper understanding of the latter. What is the meaning of such theoretical terms? How, if at all, is their reference to non-observable entities fixed? In what ways does a scientific theory contribute to the specification of the interpretation of its theoretical vocabulary? Questions of this sort are usually referred to as "the problem of theoretical terms" (e.g. $[28,37,2,25])$.

The philosophical discussion of this problem usually focuses on two related issues. The first concerns the formulation of a proper semantics for theoretical terms. More specifically, different philosophical theories are currently on the market that aim to explain a

\footnotetext{
${ }^{1}$ See [3] for an overview of the discussion and relevant literature.
} 
central semantic property of such terms, namely the fact that their meaning is usually left incomplete or undetermined in a relevant sense. Put differently, it is often the case that the reference of theoretical terms is not specified uniquely by the theory in which they occur. $^{2}$ A central topic in the literature is thus the question how to accommodate for this indefinite character of the theoretical vocabulary in the logical reconstruction of scientific theories. The second issue under discussion concerns the philosophical interpretation of different forms of theory reconstruction with respect to their ontological underpinnings. Specifically, the question here is whether a particular representation of theoretical terms commits one to a realist or non-realist account of theoretical entities such as electrons, genes, or wave functions. It is here that work on the "logic of science" in the tradition of Carnap connects with more general debates in the philosophy of science, in particular with the realism-antirealism controversy and with scientific structuralism. ${ }^{3}$

The present paper will address both issues by investigating a specific proposal made by Carnap on how to define theoretical terms. His mature work on the logical reconstruction of scientific theories - as documented in [16] and [44] - consists of two components. The first is the elimination of the theoretical vocabulary of a theory in terms of its ramsification. Roughly put, this is the reformulation of a theory - understood as a single formula expressing the conjunction of its axioms - by the substitution of its theoretical terms by existentially quantified variables. The second component consists in the reintroduction of the theoretical vocabulary by explicit definitions in the observational language supplemented by a logical epsilon operator. Intuitively speaking, these definitions pick out an arbitrary sequence of "theoretical entities" in the domain of the background language as the referents of the theoretical terms provided that the theory in question is consistent. We will dub this two-step approach the epsilon-reconstruction of scientific theories.

This paper assesses Carnap's epsilon-reconstruction of theories both from a logical and a philosophical perspective. The objective in the first part of the paper will be to give a modernized presentation of the logic underlying Carnap's approach, in particular his use of Hilbert's epsilon-operator in the definition of theoretical terms. As we will see, a closer study of this logical framework and its standard choice-functional semantics will allow us to

\footnotetext{
${ }^{2}$ For a discussion of this indefinite character of theoretical terms see, in particular, [2], [18], [25], and [43]. Carnap's work on the so-called "partial" or "indirect interpretation" of theoretical terms in [13] and [14] can be considered as an early systematic attempt to deal with this indefinite character of such terms.

${ }^{3}$ Work on this topic has so far focused mainly on the Ramsification of theories (to be described below) and its ontological commitments. See, in particular, [28], [17], and [37] for early contributions as well as [43], [20], and [24] for more recent discussions.
} 
make precise the informal talk of the indefinite or indeterminate character of such terms. The second part of the paper will then give a philosophical evaluation of the epsilonreconstruction of theories. Recent scholarship on Carnap's mature logic of science (in particular $[43,25,21])$, has considered whether his account reflects a "neutral" perspective between a realist and an instrumentalist conception of scientific theories. Moreover, it has been suggested that his account is compatible with scientific structuralism. Compare, for instance, Friedman on this point: ${ }^{4}$

[Carnap] may in fact have articulated a version of structuralism that recognizes the strengths of both instrumentalism and realism while simultaneously avoiding the philosophical "pseudoquestions" on which they appear substantively to differ. [25, p.252]

The central aim in the second part of the present paper will be to further address this philosophical conjecture. More specifically, the aim will be to see whether and if so, in what sense, Carnap's approach is compatible with a structuralist conception of scientific theories. ${ }^{5}$

Our discussion of Friedman's observation will be based on three simplifying assumptions: first, we adopt a deflationist or Lewisian understanding of the theoretical vocabulary. According to this view, theoretical terms are those terms newly introduced by a theory in question. Put differently, they are terms implicitly defined by the axioms or postulates of that theory (see [34]). In turn, the observational vocabulary consists of "old" terms whose meaning has already been specified elsewhere, for instance, in a predecessor theory. ${ }^{6}$ Adopting this understanding will allow us to sidestep the traditional debates (and

\footnotetext{
${ }^{4}$ Remarks to a similar effect can be found in [43] and [21]. Compare also Cei \& French: "Carnap's formulation leads to a structuralist treatment of the theoretical terms implying a form of multiple realisability." $[18$, p.643]

${ }^{5}$ Structuralism will be understood here primarily as an epistemic thesis about the nature of scientific theories. Roughly put, it is the view that theories investigate only the structural properties of their subject fields while remaining ignorant about qualitative or intrinsic properties of the objects considered. Such an account of scientific knowledge has a long history and traces back to work by Poincaré, Russell, Ramsey, Maxwell, and Carnap. See, in particular, [21] for an extensive investigation of the historical roots of scientific structuralism. Compare [51] and [32] for formative contributions to the modern debate.

${ }^{6}$ Compare Lewis on this understanding: "I do not understand what it is just to be a theoretical term, not of any theory in particular, as opposed to being an observational term (or a logical or mathematical term). I believe I do understand what it is to be a $T$-term: that is, a theoretical term introduced by a given theory $T$ at a given stage in the history of science. If so, then I also understand what it is to be an $O$-term: that is, any other term, one of our original terms, an old term we already understood before the new theory $T$ with its new $T$-terms was proposed." [34, p.428]
} 
philosophical problems discussed therein) on the proper demarcation of theoretical and observational terms.

Second, in contrast to the usual focus on the empirical sciences, we will restrict our attention to purely mathematical theories, in particular to axiomatic theories from abstract algebra and number theory. Carnap's epsilon-reconstruction will thus be applied to pure mathematics in this paper. As we will see, this focus will have several simplifying effects for the subsequent discussion. In particular, theoretical terms in the context of mathematics will be understood as the primitive terms of a formal language whose meaning is specified implicitly by the axioms of a particular theory. ${ }^{7}$

Third, our focus in addressing the philosophical underpinnings of Carnap's account will not be on scientific structuralism in general, but on a closely related position, namely mathematical structuralism. Roughly put, this is the view that mathematical theories study only abstract structures or structural properties of mathematical objects. An important aim in the paper will be to clarify the conceptual similarities between the epistemic structuralism associated with Carnap's logical reconstruction of theories and such a structuralist account of mathematical knowledge. Specifically, Friedman's claim will be translated into the following questions in the present paper: is Carnap's epsilon-reconstruction of theories compatible with a structuralist conception of mathematics? If so, which version of mathematical structuralism squares best with his account?

The paper will be organized as follows: Section 2 will present Carnap's epsilon-reconstruction of theories and discuss two mathematical examples from algebra and number theory. Section 3 will then turn to a more detailed logical discussion of the epsilon-logic underlying his account and as well as of a suitable choice semantics. Section 4 will discuss the specific nature of the semantic "indeterminedness" of Carnap's theoretical terms by contrasting Hilbert's $\epsilon$-terms, specifically their property of referring to objects arbitrarily, with socalled Russell indefinites. Section 5 will then discuss different versions of structuralism in the context of the philosophy of mathematics as well as their conceptual ties with scientific structuralism. We will outline a specific version of mathematical structuralism, usually labelled relative structuralism, and argue that it presents a natural philosophical framework for the epsilon-term reconstruction of mathematical theories. Finally, Section 6 will contain a brief outlook on different ways in which Carnap's reconstruction of theories might be applied to several open issues in philosophy of science.

\footnotetext{
${ }^{7}$ Consequently, old terms (in Lewis' sense) will not be relevant in the present context. See Section 2 for a further discussion of this point.
} 


\section{The epsilon-reconstruction of theories}

Carnap's own discussion of the use of Hilbert's $\epsilon$-operator for the logical reconstruction of theories remains rather sketchy and programmatic. The only two sources it is explicitly mentioned in are a short paper [16] as well as the posthumously published lecture notes titled "Theoretical concepts in science" from 1959 (see [44]). Moreover, his approach has so far found only little resonance in the subsequent literature. In particular, no scholarly attention has so far been dedicated to the possible applications of Carnap's account as well as its general philosophical significance. ${ }^{8}$ This section will set the stage for a closer discussion of the epsilon-reconstruction and show how it can be applied to mathematical theories.

Following Carnap's practice, we assume a rich object language in which both scientific theories and their Ramsey sentences can be formulated. This can be a type- or a settheoretical language. Unlike Carnap's original rich background theory which is typically a version of type-theory comprising enough mathematics, we shall confine our approach to set theory here. This has the effect that we are allowed to quantify over sets. Given that set theory is considered a (rather rich) first order theory, we say that we can express the Ramsey sentence in set theory, hence as a first-order sentence since relations are interpreted as sets. From this perspective no generality is lost. In the following, we sometimes go back and forth in our notation, and assume that no confusion should arise if one considers this in the light of these remarks.

Let $\mathcal{L}$ be a language of set theory with primitive ' $\in$ ' standing for the membership relation as its only non-logical constant. This language comes fully interpreted, that is, it describes a rich mathematical ontology, namely the cumulative universe of sets. Thus, as the interpretation of $\mathcal{L}$ we assume $\left(V, \in^{*}\right)$. An axiomatic theory $T$ is usually understood as a set of sentences, more specifically, as the set of its axioms. The language in which $T$ is formulated usually contains additional descriptive vocabulary, namely a number of "theoretical" terms $T_{1}, \ldots, T_{n}$ and possibly also a number of "old" (that is, previously defined) terms $O_{1}, \ldots, O_{m}$. These non-logical terms can be individual constants, predicates, or function symbols (of a given type and arity). Let $\mathcal{L}^{T}$ be the language with this signature, i.e. $\mathcal{L} \cup\left\{T_{1}, \ldots, T_{n} ; O_{1}, \ldots O_{m}\right\}$. This is what Carnap usually calls a theoretical language (see, e.g., [15]).

Following the standard convention, we can represent $T$ formally as a complex sentence

\footnotetext{
${ }^{8}$ See, however, [44], [18], and [34] for general discussions of Carnap's approach.
} 
$\Phi \in \mathcal{L}^{T}$ that expresses the conjunction of its axioms:

$$
\Phi\left(T_{1}, \ldots, T_{n} ; O_{1}, \ldots O_{m}\right)
$$

The Ramsey sentence of $T$ is now constructed by substituting its theoretical symbols by existentially quantified variables of the corresponding logical category: ${ }^{9}$

$$
\exists X_{1} \ldots \exists X_{n} \Phi\left(X_{1}, \ldots, X_{n}, O_{1}, \ldots O_{m}\right)
$$

Informally speaking, $\mathrm{RS}$ states that there are theoretical relations in the universe of $\mathcal{L}$-in our case the cumulative universe of sets - which have the features that the theory attributes to them. (We give two examples of the Ramsification of a theory for further illustration below.)

It has sometimes been argued in the literature that this method of Ramsification supports a non-realist or instrumentalist conception of theoretical concepts in science. However, as pointed out in [37, 28], and subsequently, by Carnap himself [17], the elimination of theoretical symbols does not effectively lead to an elimination of the reference to theoretical entities, relations, or functions, however these might be conceived. As Carnap puts it, the Ramsey-sentence "does indeed refer to theoretical entities by the use of abstract variables." [17, 963]. Thus, despite the fact that a direct reference to theoretical objects is eliminated in RS, the variables in the sentence still range over the class of such objects. ${ }^{10}$

As mentioned above, Carnap's mature work on the logic of science does not halt at this point. He suggests a two-step reconstruction of theoretical knowledge, where the first step consists in the Ramsification of a theory. The second step then consists in the subsequent reintroduction of its theoretical constants in either of two ways. Carnap's first, more well-known approach is in terms of the so-called "Carnap sentence," that is a conditional sentence of the form:

$$
\exists X_{1} \ldots \exists X_{n}\left(\Phi\left(X_{1}, \ldots, X_{n}, O_{1}, \ldots O_{m}\right)\right) \rightarrow \Phi\left(T_{1}, \ldots, T_{n}, O_{1}, \ldots O_{m}\right)
$$

\footnotetext{
${ }^{9}$ To keep the discussion simple, we will assume here that the theoretical vocabulary consists only of predicates of a specified arity. As a consequence, the existentially bound variables in the Ramsey sentence of a theory range over relations in the domain of $\mathcal{L}$.

${ }^{10} \mathrm{~A}$ central point of discussion in the literature is whether this fact commits one a realist conception of theoretical entities. For a detailed discussion of this and related issues concerning the proper interpretation of the Ramsey sentence reconstruction of theories see [43, 21, 25].
} 
Informally, this sentence expresses the fact that if RS of a theory is true, then so is the theory in its original formulation with theoretical terms. ${ }^{11}$

The second, less well-known approach to reintroduce the theoretical vocabulary of $T$ is through an explicit definition of them in the "observational" language supplemented by a logical $\epsilon$-operator. The $\epsilon$-operator added here is a logical term-forming operator that was originally introduced by Hilbert in the 1920s in his work on proof theory. Carnap applies the operator in the context of the logical reconstruction of theories in the following way: let $t=\left\langle T_{1}, \ldots, T_{n}\right\rangle$ stand for a tuple of theoretical terms of $T$. Then the explicit definition of tuple $t$ has this form:

$$
t={ }_{d f} \epsilon_{z} \exists X_{1} \ldots \exists X_{n}\left(z=\left\langle X_{1}, \ldots, X_{n}\right\rangle \wedge \Phi\left(X_{1}, \ldots, X_{n}, O_{1}, \ldots O_{m}\right)\right)
$$

Stated informally, the sequence of theoretical terms is defined here as referring to an arbitrary tuple of theoretical relations if the theory in question is satisfied, that is if its Ramsey sentence is true. The interpretation of the individual theoretical terms is then, in a second step, specified relative to this particular choice. Each $T_{i}$ (for all $1 \leq i \leq n$ ) is defined in the following way:

$$
T_{i}={ }_{d f} \epsilon_{Y} \exists X_{1} \ldots \exists X_{n}\left(t=\left\langle X_{1}, \ldots, X_{n}\right\rangle \wedge Y=X_{i}\right)
$$

The 'local' definitions of individual terms by $\left(\epsilon-\right.$ Def $\left.^{*}\right)$ are specified relative to the prior definition of the tuple of theoretical symbols. ${ }^{12}$

Two possible motivations for this reintroduction of the theoretical vocabulary in terms of these explicit definitions have been discussed in the recent literature. The first concerns Carnap's attempt to mimic informal deductive reasoning in scientific practice with implicitly defined theoretical terms. Specifically, such terms are reintroduced by (CS) or ( $\epsilon$-Def) in order to avoid the cumbersome use of existential instantiation that would be necessary

\footnotetext{
${ }^{11}$ While RS is supposed to capture the observational content of a theory, CS is usually considered to express its analytical content. See in particular [25] and [21] for further details on this point.

${ }^{12}$ The use of the epsilon-term in the definiens of Carnap's original formulation of $(\epsilon$-Def* $)$ is therefore redundant. The only relevant occurrence of an $\epsilon$-term is the one in the 'global' scheme ( $\epsilon$-Def), where a particular $\epsilon$-representative for the tuple $t$ of theoretical terms is fixed. Given this particular choice, the reference of the singular theoretical terms $T_{i}$ can also be specified more easily in terms of a projection function. Carnap was in fact aware of the redundancy of the $\epsilon$-operator in $\left(\epsilon\right.$-Def $\left.{ }^{*}\right)$. Compare his remarks on $\left(\epsilon\right.$-Def' D $^{*}$ in [16]: "Instead of the operator ' $\epsilon_{x}$ ' we could use here the customary description operator ' $\left(\iota_{x}\right)^{\prime}$ ', since the formula in square brackets fulfills the uniqueness condition with respect to ' $x$ '. [16, p.161].
} 
if the logical reconstruction of a theory consisted only of its Ramsification. ${ }^{13}$ The second possible motivation for introducing $t$-terms through of explicit definitions is, as Psillos has pointed out, Carnap's wish to give a non-holistic or "atomistic" specification of such terms (see [44]).

Our focus in this paper will be less on these general conceptual motivations for the $\epsilon$-reconstruction but rather on its application in the context of mathematics. We give two examples of mathematical theories to further illustrate Carnap's approach. Both will be used as toy examples in the philosophical discussion in the subsequent sections. Before turning to them, note again that a Lewisian account of theoretical terms is adopted here. This is to say that theoretical terms are understood here as those terms newly introduced by a theory in question. According to Lewis, such terms do not have pre-established meaning or interpretation. Rather, their meaning is specified indirectly or implicitly through the postulates of the theory in which they are mentioned [34, p.429].

Applied to the context of pure mathematics, theoretical terms are precisely the primitive non-logical constants of a language that are implicitly defined by the axioms of a particular theory. Since mathematical theories are treated in isolation here, there are no "old" but only theoretical terms to consider. ${ }^{14}$ Let us consider two examples to further illustrate this approach: The first example comes from abstract algebra and concerns the theory of a simple type of algebraic structures, namely of monoids:

Example 1 (Monoids) A monoid is a triple $\langle G, \circ, e\rangle$, where $G$ is a set, $e \in G$, and $\circ$ is a binary operation $G \times G \rightarrow G$ that satisfies two axioms:

(M1) $\forall x, y, z \in G:(x \circ y) \circ z=x \circ(y \circ z)$

(M2) $\forall x \in G: e \circ x=x \circ e=x$

\footnotetext{
${ }^{13}$ See, for instance, [24]. Friedman puts this point as follows: "For the Carnap sentence is now seen to take over the role of precisely existential instantiation from the Ramsey-sentence, and it then allows us to proceed with ordinary mathematical reasoning in the style of Hilbert without worrying about cumbersome restrictions on existential variables in natural deduction. Whereas existential instantiation, of course, is not a logically valid inference, the Carnap sentence (...), taken as a non-logical axiom of $T$, is now seen, nonetheless, as an analytic postulate - a conventional choice of (constant) names arbitrarily given to a sequence of values of the variables (...), which, by the Ramsey-sentence, must (synthetically) exist. " [24, p.397]

${ }^{14}$ It is possible, however, to speak also of old terms in the context of mathematics, at least if these terms are understood in Lewis's sense. Such terms become relevant in cases where extensions of a mathematical theory are considered. Consider the example of Robinson arithmetic presented below: Axiom system (Q1Q7) implicitly defines the terms $S,+, *$. Thus, relative to theory (Q1-Q7), these count as theoretical terms in our understanding. However, relative to the extended axiomatic theory (Q1-Q8), expressions $S,+, *$ can also be viewed as old terms, i.e. as already defined terms. This issue will not be pursued here any further.
} 
The Ramsey sentence of the theory $\operatorname{Mon}[G, \circ, e]$ is:

$$
\begin{array}{r}
\exists X \exists f \exists v \forall x \forall y \forall z(X(x) \wedge X(y) \wedge X(z) \rightarrow(f(f(x, y), z)=f(x, f(y, z)) \wedge \\
f(v, x)=f(x, v)=v))
\end{array}
$$

The $\epsilon$-definition of $t=\langle G, \circ, e\rangle$ then is:

$$
t={ }_{d f} \epsilon_{z} \exists X \exists f \exists v(z=\langle X, f, v\rangle \wedge \operatorname{Mon}[X, f, v])
$$

The second example is from number theory and concerns those number systems satisfying a weakened version of Peano arithmetic, namely Robinson arithmetic.

Example 2 (Robinson arithmetic (Q)) Let $\mathcal{L}_{A}$ be the language of arithmetic. $Q$, aka Robinson Arithmetic is axiomatized as follows: ${ }^{15}$

(Q1) $S(x) \neq 0$

(Q2) $S(x)=S(y) \rightarrow x=y$

(Q3) $x \neq 0 \rightarrow \exists y(x=S y)$

(Q4) $x+0=x$

(Q5) $(x+S y)=S(x+y)$

(Q6) $(x * 0=0)$

(Q7) $(x * S y)=(x * y)+x$

(Q8) $x \leq y \leftrightarrow \exists z(x+z)=y$

Schematically, the conjunction of (Q1)-(Q8) can be represented as $\mathrm{Q}[\mathrm{S},+, *, \leq]$. The Ramseysentence of $\mathrm{Q}[\mathrm{S},+, *, \leq]$ is:

$$
\exists f^{1} \exists f^{2} \exists f^{3} \exists r^{4} \mathrm{Q}\left[f^{1}, f^{2}, f^{3}, r^{4}\right]
$$

The sequence of theoretical terms $t=\langle\mathrm{S},+, *, \leq\rangle$ is then defined as:

$$
t=\epsilon_{z} \exists f^{1} \exists f^{2} \exists f^{3} \exists r^{4}\left(z=\left\langle f^{1}, f^{2}, f^{3}, r^{4}\right\rangle \wedge \mathrm{Q}\left[f^{1}, f^{2}, f^{3}, r^{4}\right]\right)
$$

Two points of commentary concerning these examples are in order here. First, notice that the respective Ramsey sentence simply expresses the consistency of the theory in question here, viz. the fact that there exists at least one model that satisfies it. As we said, the $\epsilon$-term definition then picks out one object in the model class in question and specifies the interpretation of the primitive terms relative to this choice. In the case of monoids, this

\footnotetext{
${ }^{15}$ See, e.g., [27, p.28].
} 
$\epsilon$-representative can be any structured set living in the cumulative hierarchy of sets $V$ that satisfies the two monoid axioms, for instance $\langle\mathbb{N},+, 0\rangle$ or $\langle\mathbb{R}, \times, 1\rangle$. The reference of the primitive terms ' $G$ ', 'o', ' $e$ ' is then specified relative to either one of these structures.

Second, a point concerning the ontological underpinnings of this logical reconstruction: it should be clear that Carnap's approach does not lend support to a nominalist account of mathematics. In particular, it presupposes a rich mathematical background ontology, in our case, the cumulative universe of sets. Given these strong set-theoretic assumptions, it is not clear, at least prima facie, how Carnap's approach should at the same time be compatible with a structuralist conception of mathematical theories. In order to address this connection with structuralism, we have to get more specific about the underlying logic of Carnap's approach, in particular his use of the $\epsilon$-operator and its standard semantic interpretation.

\section{Epsilon terms and their choice semantics}

The key component in Carnap's logical reconstruction of theories is Hilbert's $\epsilon$-operator. ${ }^{16}$ This Section will present the logic of this operator as well as its standard semantic interpretation. As mentioned above, the $\epsilon$-operator functions as a logical term-forming operator: if $A(x)$ is a formula with $x$ occurring as free variable in it, then $\epsilon_{x} A(x)$ is a term where all occurrences of $x$ are bound. ${ }^{17}$

Deductive systems describing the logical behaviour of such epsilon terms, so-called Epsilon Calculi (EC), usually consist of two central axioms (in addition to the standard axioms and deduction rules of first-order logic):

$$
\begin{array}{rr}
A(t) \rightarrow A\left(\epsilon_{x} A(x)\right) & \text { (Critical formulas) } \\
\forall x(A(x) \leftrightarrow B(x)) \rightarrow \epsilon_{x} A(x)=\epsilon_{x} B(x) & \text { (Extensionality) }
\end{array}
$$

The extensionality axiom states that if two formulas are equivalent, then their corresponding $\epsilon$-representatives are identical. Given these two axioms, the standard first-order quan-

\footnotetext{
${ }^{16}$ See [52] for a detailed history of the epsilon logic. Compare [33], [53], and [39] for modern presentations of the epsilon calculus and its semantics.

${ }^{17}$ Given a second-order or type-theoretic language, "higher-order" epsilon terms can be constructed in a similar way. For instance, let $A(X)$ be a formula with a free $n$-ary relation variable $X$. The term $\epsilon_{X} A(X)$ will pick out an arbitrary $n$-ary relation on the domain of the model relative to which statement $A$ is true. Epsilon terms built from function variables of any arity can be constructed in a similar way.
} 
tifiers can be defined explicitly in the following way:

$$
\begin{aligned}
\exists x A(x) & : \leftrightarrow A\left(\epsilon_{x} A(x)\right) \\
\forall x A(x) & : \leftrightarrow A\left(\epsilon_{x} \neg A(x)\right)
\end{aligned}
$$

As Carnap has pointed out in [44] and [16], (Def $\exists$ ) together with his definitional scheme for $t$-terms allows one to deduce the Carnap sentence of a theory. Thus, he was able to show logically, i.e. in terms of a simple argument given in EC, the equivalence of his two logical reconstructions of theories, namely of (1) RS and CS and (2) RS and ( $\epsilon$-Def).

It is important to note, however, that the main conceptual motivation for the introduction of $\epsilon$-terms in Carnap's reconstruction of theories does not lie in their inferential use but rather in their semantics. More specifically, it concerns a central intuition about a semantic feature of the $\epsilon$-operator, namely a kind of referential indeterminacy of the terms constructible from it. Compare the following remark made by Carnap on the special nature of $\epsilon$-terms as "indeterminate constants":

(...) the symbol ' $\epsilon$ ' was intentionally introduced by Hilbert as an indeterminate constant. Its meaning is specified by the axioms (1) and (2) only to the extent that any non-empty set has exactly one representative and that this representative is an element of the set. If the set has more than one element, then nothing is said, either officially or unofficially, as to which of the elements is the representative. $[16, \text { pp.162-163 }]^{18}$

Unfortunately, Carnap never gave an explicit specification of the semantics of the $\epsilon$-operator and of the terms constructible from it in his work. Nevertheless, there exists a natural explication of his intuitions about the "incomplete" or "indeterminate meaning" of such expressions in terms of a choice-functional semantics, that is a semantic interpretation of epsilon terms based on choice functions. ${ }^{19}$

A choice semantics for an extensional epsilon logic is usually characterized in the following way: ${ }^{20}$ an interpretation $\mathfrak{M}$ of the language $\mathcal{L}_{\epsilon}$ has the form $\langle D, I\rangle$, where $D$ is a domain

\footnotetext{
${ }^{18}$ Compare also the following related remark: “(...) the Hilbert $\epsilon$-operator belongs to a small class (...) of logical constants of a very particular kind. I will call them indeterminate. They are such that their meaning is not completely specified." [44, p.171]

${ }^{19}$ It should be noted here that Carnap was aware of the possibility of such a choice-functional interpretation of epsilon logic. He refers in [16] to Asser's paper "Theorie der logischen Auswahlfunktionen" [4] were a first systematic treatment of different choice semantics for the epsilon calculus is given.

${ }^{20}$ We follow closely here Zach's presentation of a choice semantics given in [53]. For a different semantic
} 
and $I$ an interpretation function for the nonlogical vocabulary. We say that $s: \operatorname{Var} \rightarrow D$ is an assignment function on $\mathfrak{M}$. The $\epsilon$-operator is interpreted by an extensional choice function $\delta: \wp(D) \rightarrow D$ such that, for any $X \subseteq D$ :

$$
\delta(X)= \begin{cases}x \in X, & \text { if } X \neq \emptyset \\ x \in D & \text { otherwise }\end{cases}
$$

The choice function picks out a "representative" object for any non-empty set and an arbitrary object from domain $D$ if the set in question is empty. ${ }^{21}$ Given this framework, the reference of $\epsilon$-terms can be specified relative to structure $\mathfrak{M}$, assignment function $s$, and choice function $\delta$ on $\mathfrak{M}$ based on the following valuation rule: ${ }^{22}$

$$
\begin{aligned}
& v a l^{\mathfrak{M}, \delta, s}\left(\epsilon_{x} A(x)\right)=\delta\left(v a l^{\mathfrak{M}, s}(A(x))\right) \\
& =\delta(\{d \in D \mid \mathfrak{M}, s[x / d] \models A(x)\}) .
\end{aligned}
$$

Stated less formally, the rule states that the referent of an $\epsilon$-term formed from formula $A$ is the element that the choice function picks from the subset of domain $D$ defined by $A$.

This choice-functional semantics for $\epsilon$-terms gives us a natural explication of the informal semantic treatment of theoretical terms implicit in Carnap's logical reconstruction of theories. To see this, consider again the theory of monoids presented above. As we saw, the definition(s) of its primitive terms can be formulated in $\mathcal{L}_{\epsilon}$, that is, in a first-order language of set theory supplemented by an $\epsilon$-operator. The semantic evaluation of the $\epsilon$-term definition of the primitives ' $G$ ', ' $O$ ', and ' $e$ ' can then be specified relative to a given choice function. What the $\epsilon$-term definition does in this case is to pick out one object from the class of monoids. The reference relation for theoretical terms is thus directly modeled by the choice-functional interpretation of their defining $\epsilon$-terms. For instance, relative to

account, see, e.g., [33].

${ }^{21}$ Given that our chosen background theory is set theory here, we can formulate choice functions as a first-order condition in the above sense without any loss of generality. Nevertheless, choice functions for higher-order epsilon terms can, in principle, be constructed in a similar way. Consider again the epsilon term $\epsilon_{X} A(X)$ described in footnote 17. A suitable choice function for it is a function of the form $\delta: \wp\left(\wp\left(D^{n}\right)\right) \rightarrow \wp\left(D^{n}\right)$ such that, for any set of relations $\mathbf{R} \subseteq \wp\left(D^{n}\right)$, we have:

${ }^{22}$ See again [53].

$$
\delta(\mathbf{R})= \begin{cases}X \in \mathbf{R}, & \text { if } \mathbf{R} \neq \emptyset \\ X \in \wp\left(D^{n}\right) & \text { otherwise }\end{cases}
$$


one such function, the extension of ' $G$ ', 'o', and ' $e$ ' might be specified as $\mathbb{N},+$, and 0 respectively. Relative to another choice function, it will be specified as $\mathbb{R}, \times$, and 1 . As we will see below, the reference of these primitive terms remains indeterminate, however, in the sense that there is no principled way to choose one such interpretation over the other.

This choice semantics for $\epsilon$-terms not only provides a precise account of the reference of theoretical terms that nicely corresponds to Carnap's informal remarks. It also allows a general explication of the semantics of theoretical sentences, that is sentences containing such terms. This explication comes in two steps: First, a translation function has to be specified that maps theoretical sentences to their $\epsilon$-translations, i.e. to those sentences resulting from the systematic substitution of $t$-terms by the $\epsilon$-terms defining them. More formally, let $A$ be a sentence in a theoretical language $\mathcal{L}^{T}$ with signature $\left\{t_{1}, \ldots, t_{n}\right\}$. Then, given Carnap's method of defining the theoretical terms via $\epsilon$-terms, there is a function $f: \mathcal{L}^{T} \rightarrow \mathcal{L}_{\epsilon}$ that maps every theoretical sentence $A$ to a sentence $A^{*}$ such that each occurrence of $t_{i}$ (for every $i \in n$ ) in $A$ is substituted by its defining $\epsilon$-term. Second, the resulting $\epsilon$-translate $A^{*}$ can then be evaluated in terms of the choice-functional semantics in the way presented above. More specifically, we can distinguish between two semantic notions of relevance for theoretical sentence:

Explication 1 (Semantics of theoretical sentences) Let $A$ be a theoretical sentence, $A^{*}$ its $\epsilon$-translation in $\mathcal{L}_{\epsilon}$, and $\mathfrak{M}$ an interpretation of $\mathcal{L}_{\epsilon}$. We say that

1. A is true in $\mathfrak{M}$ iff there exists a choice function $\delta$ on $\mathfrak{M}$ such that $\mathfrak{M}, \delta \models A^{*}$;

2. A is universally true (or valid) in $\mathfrak{M}$ iff for every choice function $\delta$ on $\mathfrak{M}$, we have $\mathfrak{M}, \delta \mid=A^{*}$.

This choice-functional explication of the informal semantics of theoretical sentences can be further clarified by looking more closely at the simple model theory underlying it. According to the semantic conception of theories, a theory $T$ (expressed by the complex sentence $\Phi)$ can also be characterized as a class of models in the following way:

$$
\operatorname{Mod}^{t} \Phi:=\{\mathfrak{M}: \mathfrak{M} \text { is a } t \text {-structure } \wedge \mathfrak{M} \models \Phi\}
$$

where $t$ stands for the theoretical signature $\left\{t_{1}, \ldots, t_{n}\right\}$ of the language in which $\Phi$ is formulated. In Carnap's reconstruction of theory $T$, a particular choice function interpreting the $\epsilon$-operator picks out one model from such a class and specifies the interpretation of the 
theoretical vocabulary relative to this choice. Different choice functions pick out different objects from the same model class. The notions of truth and universal truth of theoretical sentences are then evaluated in terms of the possible choice functions on a theory's model class. $^{23}$

Let us give two simple examples of mathematical sentences to illustrate this account. Consider first a sentence expressible in terms of the vocabulary of monoids presented in Example 1. The sentence in question states the existence of inverses in algebraic structures:

$$
\forall x \in G \exists y \in G: x \circ y=y \circ x=e
$$

This sentence is true of some monoids, namely those forming a group. However, it is certainly not true of all monoids. (Our above example of structure $\langle\mathbb{N},+, 0\rangle$ is a case in point here.) The fact that sentence $\psi$ is realizable can be formally established by translating it into its corresponding $\epsilon$-sentence. This will be a "purely" logical sentence in language $\mathcal{L}_{\epsilon}$ that can be semantically evaluated in the above sense. The observed fact that $\psi$ is true in some but not all monoids is captured by the fact that there exists at least one choice function $\delta$ on the intended set-theoretic universe $V$ of language $\mathcal{L}_{\epsilon}$ that assigns values to the $\epsilon$-terms defining ' $G$ ', 'o', and ' $e$ ' such that $V, \delta \models \psi^{*}{ }^{24}$

Consider now the simple arithmetical sentence ' $2+2=4$ '. It can easily be translated into a sentence containing only the primitive arithmetical terms ' 0 ', ' + ', and ' $S$ ' presented in Example 2, namely:

$$
S(S(0))+S(S(0))=S(S(S(S(0))))
$$

This is a simple theorem of Q, i.e. it is logically provable from the axioms listed in Example 2. As a consequence, it is also valid, that is, it holds in all number structures satisfying Q. This semantic fact of the validity of $\varphi$ can again be represented formally in the above

\footnotetext{
${ }^{23}$ Note that the present explication of theoretical truth is based on a variable choice interpretation of the $\epsilon$-operator: $\epsilon$-terms (and thus sentences containing them) are evaluated relative to a all possible choice functions for a given model. The question whether such a flexible choice semantics is in conformity with Carnap's original understanding of epsilon terms will be addressed in a separate paper.

${ }^{24}$ It should be emphasized here that this semantic evaluation of a sentence like $\psi$ is, in an important sense, theory relative. In Carnap's approach, the $t$-terms are defined relative to a particular theory (expressed in the definiens of the $\epsilon$-term definition). It follows that the same terms are defined differently relative to different theories. For instance, the same algebraic signature can be defined relative to the theory of monoids or to group theory. These different theoretical contexts have direct implications for the semantic evaluation of the sentences expressed in terms of these theoretical terms. Sentence $\psi$ is, as we saw, satisfied relative to the background theory of monoids, but obviously valid relative to the theory of groups.
} 
way by translating $\varphi$ into a sentence of $\mathcal{L}_{\epsilon}$ based on Carnap's $\epsilon$-term definitions. As can be shown, the $\epsilon$-translation will turn out true in universe $V$ for any possible choice function assigning values to the $\epsilon$-terms standing for the primitives ' 0 ', ' + ', and ' $S$ '.

The present account turns out to capture closely Carnap's general views on the crucial semantic feature of theoretical terms, namely their "indefinite" or "indeterminate" nature. ${ }^{25}$ In the following Section, we further specify the particular status of theoretical vocabulary as semantically indeterminate by looking at the special type of reference of their corresponding epsilon terms.

\section{Indefinites and arbitrary reference}

The epsilon-operator was introduced in Carnap's logical framework to allow for an explicit definition of the theoretical terminology of a scientific theory. As we saw, a central motivation for this was to reintroduce theoretical terms after their elimination by Ramsification in order to describe more adequately the deductive inferences in actual science. A second, equally important motivation was to capture in precise terms a certain indeterminacy or non-uniqueness of reference that is typical for such terms. Compare again Carnap on this second semantic point:

The postulates TC [of a theory] are intended by the scientist who constructs the system to specify the meaning of ' $t$ ' to just this extent: if there is an entity satisfying the postulates, then ' $t$ ' is to be understood as denoting one such entity. Therefore the definition (...) gives to the indeterminate constant ' $t$ ' just the intended meaning with just the intended degree of indeterminacy. [16, p.163, emphasis added]

As we saw, EC and its choice-function semantics provide a natural logical framework for modeling this semantic indeterminacy. Nevertheless, in order to connect Carnap's logical approach with scientific structuralism, more needs to be said about the philosophical interpretation of the specific kind of semantic indeterminacy of $\epsilon$-terms. How can their specific mode of reference be characterized more generally in philosophical terms?

\footnotetext{
${ }^{25}$ See in particular [14]. As has been pointed out in [44] and [2], this fact of the "non-uniqueness" of the interpretation of theoretical terms distinguishes Carnap's $\epsilon$-definition from Lewis' explicit definition of such terms by use of a logical $\iota$-operator [34].
} 
Interestingly, $\epsilon$-terms are often described as indefinite descriptions in the philosophical literature. ${ }^{26}$ This is a natural claim to make given that they are usually paraphrased in terms of indefinite noun phrases: $\epsilon_{x} A(x)$ is standardly read as "an $A$, if there exists one". Moreover, in recent work in linguistics and semantic theory, $\epsilon$-terms are frequently used for the logical representation of dependent indefinite phrases of the form "an $A$ is a $B$ " (e.g. [50]). Statements of this form are also ubiquitous in scientific reasoning and in mathematical reasoning in particular. Consider, for instance, stipulative sentences with indefinites such as "Let $G$ be a complete graph" or "Let $A$ be a cyclic group of order $n$ " which usually stand at the beginning of a mathematical proof. According to the above view, their logical structure is best represented in the language of the epsilon calculus by $B\left(\epsilon_{x} A(x)\right)$. But precisely what kind of indefinites are $\epsilon$-terms? And, if descriptions constructed from them are understood in their "referential" sense, what kind of reference is typically associated with them?

In order to address these questions, it seems helpful to compare the indefinites expressed by $\epsilon$-terms - let us dub them Hilbert indefinites - with the standard Russellian account of indefinite or ambiguous descriptions. ${ }^{27}$ Roughly put, an ambiguous description in the latter sense is the occurrence of an indefinite phrase "an $A$ " in a particular sentential context $B$, viz. "an $A$ is a $B$ ". The standard formalization of this in first-order logic is:

$$
\exists x(A(x) \wedge B(x))
$$

where both $A$ and $B$ are unary predicates or formulas. ${ }^{28}$ As is shown in [26], Russell's indefinites can alternatively be expressed in terms of an term-forming operator that is in several ways similar to Hilbert's $\epsilon$-operator. For a given formula $A$ with $x$ occurring free in it, let $\rho_{x}(A(x))$ be a term standing for "an $x$, such that $x$ has $A$ ". This $\rho$-operator is defined in the following way:

$$
B\left(\rho_{x}(A(x))\right): \leftrightarrow \exists x(A(x) \wedge B(x))
$$

Given a system $\mathfrak{M}$, let $\mathcal{A} \subseteq \operatorname{dom}(\mathfrak{M})$ be the set of objects defined by formula $A$, and let $\mathcal{B} \subseteq \operatorname{dom}(\mathfrak{M})$ be the set defined by formula $B$. Intuitively speaking, the operator $\rho$ picks

\footnotetext{
${ }^{26}$ Psillos, for instance, states that: "(...) in a sense, the $\epsilon$-operator characterises an indefinite description (...)." $[44,157]$

${ }^{27}$ For Russell's remarks on ambiguous descriptions as opposed to definite descriptions, see his [47].

${ }^{28}$ An indefinite description can thus be constructed from the definite description "the $A$ is a $B$ ", expressed by $\exists x(A(x) \wedge \forall y(A(y) \rightarrow x=y) \wedge B(x))$, simply by dropping the uniqueness clause, that is the claim that the description fixes the reference to one particular object in the domain.
} 
out one element in $\mathcal{A}$ that is also in $\mathcal{B}$ (assuming that their intersection is non-empty). The central conceptual idea underlying this Russellian account of indefinites is a kind of semantic context dependency, that is the fact that the specification of an $A$-representative picked out by the operator depends on the particular sentential context in which formula $A$ occurs. In terms of the informal semantics underlying the $\rho$-operator, this point is given by the constraint that the selection of a particular $\rho$-representative of set $\mathcal{A}$ is specified only relative to a given 'context' set $\mathcal{B}$ in which it also occurs. Thus, in a slogan, one can say that the reference of a given term is a function of its particular sentential context. ${ }^{29}$

How are Hilbert's and Russell's accounts of indefinite descriptions related? This question can be addressed in a precise way by comparing the two underlying logics and their respective term-forming operators. It can be shown by a simple argument that the two notions of indefiniteness do not coincide. More specifically, for any two first-order formulas $A, B$, we can show that:

$$
B\left(\epsilon_{x} A(x)\right) \leftrightarrow B\left(\rho_{x}(A(x))\right)
$$

Proof sketch: To show that the left-to-right implication fails, consider a model $\mathfrak{M}$ where $\mathcal{A}=\emptyset$ as well as a choice-function $\delta$ interpreting the $\epsilon$-operator such that $\delta(\mathcal{A})=x \in \mathcal{B}$. Relative to $\mathfrak{M}$ and $\delta$, the antecedent will turn out true. However, the consequent must be false, since any possible selection function interpreting the $\rho$-operator will give an element in the domain outside $\mathcal{B}$, since $\mathcal{A} \cap \mathcal{B}=\emptyset$. In order to show the right-to-left direction to be non-valid, consider a model where $\mathcal{A} \cap \mathcal{B} \neq \emptyset$ and $\mathcal{A} \nsubseteq \mathcal{B}$. Consider a choice function interpreting the $\epsilon$-operator such that $\delta(\mathcal{A})=x \notin \mathcal{B}$. The right hand side formula will turn out true relative to this model (and any possible selection function interpreting the $\rho$-operator). However, this left hand side formula will be false relative to the particular choice function.

This result states, in precise terms, that Hilbert indefinites are not identical to Russell indefinites. A closer diagnosis shows that the reason for this difference is genuinely semantic in nature. It concerns the fact that the reference of a $\rho$-term is not specified in isolation,

\footnotetext{
${ }^{29}$ More formally and in analogy with the choice semantics for the $\epsilon$-operator, we can specify a functional interpretation of the $\rho$-operator in the following way. For the above example, the selection function will have the form:

$$
\Psi(\mathcal{A})= \begin{cases}x \in \mathcal{A} \cap \mathcal{B} & \mathcal{A} \cap \mathcal{B} \neq \emptyset \\ x \in D \backslash A \cup B & \text { otherwise }\end{cases}
$$

Thus, for a given formula $A$ and a given context $B$, the function picks out an element in the intersection of the sets $\mathcal{A}$ and $\mathcal{B}$, if this set is nonempty, and an arbitrary element in the domain otherwise.
} 
but only relative to a given subset of the domain defined by a formula in which the term occurs. The specific referential nature of such terms is such that they can refer to one thing in a given (sentential) context, and to a different thing in another context. This context dependency is clearly missing in the choice semantics for the $\epsilon$-logic outlined in Section 3. Given a specific choice-functional interpretation of an epsilon term, its semantic value remains invariant or stable under all changes of sentential contexts here. ${ }^{30}$

Where does this leave us with respect to the philosophical evaluation of free choice indefinites presented by $\epsilon$-terms? As we saw, epsilon terms differ from Russell's $\rho$-terms in at least two respects. First, indefiniteness in the former case does not mean semantic indeterminacy in absence of context. Second, it also does not merely mean non-uniqueness of reference as opposed to the standard account of definite descriptions. What seems to be genuinely characteristic for $\epsilon$-terms is a specific mode of reference associated with them, that is, a specific way of referring to objects in the domain in question. This is sometimes termed arbitrary reference in the philosophy of language. ${ }^{31}$

Typical cases of arbitrary reference are usually discussed in connection with so-called "instantial terms" in deductive reasoning, in particular, with the use of such terms in the $(\exists$-Elimination) and the $(\forall$-Introduction)-rules in natural deduction. Other examples concern the use of "arbitrary names" in mathematical reasoning, in particular the use of indefinite terms in stipulative statements such as "Let $G$ be a graph of order $n$." already mentioned above. ${ }^{32}$ Breckenridge \& Magidor give a general characterization of the kind of reference typical in such cases:

Arbitrary Reference (AR): It is possible to fix the reference of an expression arbitrarily. When we do so, the expression receives its ordinary kind of semanticvalue, though we do not and cannot know which value in particular it receives. $[10$, p.378]

Two points of specification are in order here: first, terms that refer arbitrarily in this sense do refer to particular objects. That is, their reference is not undetermined in the

\footnotetext{
${ }^{30}$ While Russell's indefinites cannot be expressed in the language of EC, it turns out that they can be formulated in a natural extension of it, namely in a language of indexed epsilon terms [50, 38]. Another possible connection between the $\rho$-operator and epsilon logic concerns Bell's work on the so-called "dependent" epsilon terms in the context of intuitionistic logic [6]. A closer comparison between the logic of the indexed $\epsilon$-operator, Bell's work on dependent choice, and the Russellian $\rho$-operator will be given in a separate paper.

${ }^{31}$ See, in particular, [10] for the most extensive discussion of arbitrary reference in the literature.

${ }^{32}$ See [36] for an explicit discussion of arbitrary reference in the context of mathematics. Compare also [10] for an interesting discussion of instantial reasoning in geometrical reasoning.
} 
sense that they fail to denote individual objects. This point distinguishes the present ARaccount from other explanations of the semantic behaviour of instantial terms, in particular the "quantificational" account of such terms. ${ }^{33}$ Second, as is pointed out in [10], AR does not mean that these terms refer to non-ordinary or arbitrary objects in the domain in the sense of Fine's theory of arbitrary objects (e.g. [22]). ${ }^{34}$ What makes instantial terms "arbitrary" is the fact that the reference relation itself is left undetermined or unspecified. As Breckenridge \& Magidor point out, what distinguishes AR from Fine's account is the difference between referring arbitrarily to ordinary and particular objects in the domain and referring to arbitrary objects (in Fine's sense of the term).

It should be clear at this point that Hilbert's $\epsilon$-terms refer to objects in precisely this way. AR can therefore be seen as a natural philosophical description of the kind of reference specified by the choice functional semantics for such terms. Note in particular that $\epsilon$-terms meet the two characteristic features of AR just described: relative to a given choice structure, an $\epsilon$-term denotes a singular object in the domain. Thus, the mode of reference here is not "quantificational" or plural in nature. Moreover, epsilon terms do not presuppose a separate domain of nonstandard, arbitrary objects as in Fine's account but models with a uniform domain. ${ }^{35}$ What makes the semantic framework nonstandard in the sense of $\mathrm{AR}$ is the fact that the choice functions specifying the selection made by an $\epsilon$-operator guarantee that this selection is completely arbitrary. Thus, the most suitable translation of $B\left(\epsilon_{x}(A(x))\right)$ into colloquial English is "an arbitrary $A$ is a $B$ ".

We can conclude from this that "arbitrary reference" is the defining and philosophically relevant semantic feature of the epsilon logic. Hilbert's $\epsilon$-terms belong to a specific type of indefinite descriptions that behave semantically like arbitrary reference names, i.e. as singular expressions that refer to objects arbitrarily. As we will see in the next section, it is precisely this feature of arbitrary reference that also connects Carnap's explicit use of $\epsilon$-terms in his logical reconstruction of theories with a structuralist understanding of the latter. More specifically, we will show that the epsilon-reconstruction gives a natural

\footnotetext{
${ }^{33}$ According to the latter view, instantial terms are best explained not as denoting particular objects but rather as ranging over a class of relevant objects just as variables do. Thus, terms or names are really variables in disguise, that is plurally referring expressions. For a detailed presentation of such a position, see, in particular, [31]. Compare [10] for a detailed critique of this approach as well as a comparison of the different approaches to instantial terms.

${ }^{34}$ Fine's account holds that in contexts of logical and mathematical reasoning, instantial terms can be taken to refer not to individual objects, but to objects of a different kind, namely arbitrary objects.

${ }^{35}$ Thus, one can say that while Fine assumes a classical semantics but a nonclassical ontology, the epsilonlogic is based on a nonclassical choice semantics, but requires only a standard ontology of objects.
} 
explication of the informal semantics of mathematical terms if one adopts a particular version of mathematical structuralism.

\section{Eliminative structuralism}

As mentioned in the Introduction, it has recently been observed by Friedman and others that Carnap's logical reconstruction of theories is compatible with scientific structuralism. Compare, for instance, Demopoulos' general characterization of a "structuralist thesis" underlying Carnap's work:

The theoretical component of what our theories express in wholly captured by statements which depend only on the logical category of their constituent concepts. [21, p.161]

The central idea expressed here is that the theoretical - that is, the non-observationalcontent of a scientific theory is purely structural in character. Moreover, this structural content can be expressed by the theory's Ramsey sentence in the way outlined in Section 2. The structuralism described here is similar to the epistemic structural realism first introduced in [51], that is, the view that all we know about the physical world is structural in character. Nevertheless, Demopoulos' account differs from standard versions of epistemic structural realism in his focus on what makes theoretical knowledge structural in character. This is the fact that the interpretation of the theoretical vocabulary is usually left undetermined by a scientific theory. Thus, in his words, "apart from their logical category, nothing is assumed about the meaning of [a theory's theoretical] terms" [21, p.163].

It is precisely this generality of theoretical knowledge that connects Demopoulos' (and arguably also Friedman's) scientific structuralism with a structuralist account of mathematics. Specifically, we can see that a similar generality has also been ascribed to mathematical knowledge in recent debates on mathematical structuralism. ${ }^{36}$ This is, roughly put, the view that mathematical theories studies only the abstract structures of structural

\footnotetext{
${ }^{36}$ Interestingly, Demolopous describes the "structuralist thesis" in direct comparison with a central method in modern structural mathematics, namely Hilbert's formal axiomatics:

The structuralist thesis is a simple extrapolation from Hilbert's understanding of the essential generality of mathematical theories. Hilbert argued that the proper formulation of a mathematical theory should not be constrained by the demand that it preserve[s] preconceptions regarding the nature of the theory's primitive notions. [21, p.82]
} 
properties of object domains. For instance, Robinson arithmetic does not investigate particular number systems, but rather their shared abstract structure described by the axioms of Q. The central philosophical motivation for such a structuralist account is to explain so-called "Benacerraf phenomena" in mathematics. Roughly put, these are mathematical objects - first described in Benacerraf's influential article "What numbers could not be" [7] — that can be defined or interpreted in different ways. Moreover, the theory describing these objects gives us no systematic way to decide on which of these interpretations is the correct one. Thus, it leaves systematically undetermined the true nature of the mathematical objects it talks about. Benacerraf's main example to illustrate this point concerns the reduction of natural numbers to sets: the number ' 2 ' can be defined set-theoretically either as the von Neumann set ' $\{\emptyset,\{\emptyset\}\}$ ' or as the Zermelo set ' $\{\{\emptyset\}\}$ '. Any number theory at hand, for instance $Q$, remains ignorant as to which of the two set-theoretic representations is actually the correct one. It tells us only about the relational or structural properties of number ' 2 ' with respect to the other numbers in a given number system.

The ontological indifference or indeterminacy expressed in such examples has generated a number of different and partly incompatible versions of structuralism. More specifically, one can say that Benacerraf's observation has led to two distinct ways to think of the structural content of mathematical theories. The first one are so-called non-eliminative theories, for instance Shapiro's ante-rem structuralism or Resnik's theory of mathematical patterns $([46],[48])$. What is typical for these accounts is that they propose a realist picture of abstract structures. Structures are conceived here as abstract objects, for instance as structured Platonic universals that can be instantiated by "concrete" mathematical systems. ${ }^{37}$ The second camp consists of eliminative theories of structuralism. ${ }^{38}$ Such accounts are typically anti-realist or nominalist in the question concerning the existence of abstract structures. Thus, while these theories also propose an explanation of Benacerraf phenomena in mathematics, this does not depend on an additional mathematical ontology of structural objects. Moreover, non-eliminative accounts usually defend the view that mathematicians' talk about abstract structures should not to be taken literally or at face value, but rather as a way of abstracting from the systems of a theory in question.

\footnotetext{
${ }^{37}$ See, e.g., [48], [46], and [35] for different versions of such a non-eliminative position. There are interesting parallels between non-eliminative mathematical structuralism and different versions of ontic structural realism discussed in philosophy of science. Compare, e.g., [12] and [23] for a closer discussion. Compare also [9] for a more general comparison of mathematical and scientific structuralism.

${ }^{38}$ Compare [45] for a more detailed comparison of the two approaches.
} 
In the present context, we will briefly outline two eliminative approaches in mathematical structuralism that are closely in line with Demopoulos' more general description of the structuralist thesis for theoretical knowledge. Moreover, we will argue that one of them fits particularly well with Carnap's epsilon-reconstruction of theories. To see this, our focus will be on the general implications of these eliminativist accounts for the semantics of mathematical discourse, and more specifically, for the mode of reference of mathematical terms. Structuralism today mainly comprises different theories about the ontology of mathematics, that is the metaphysical nature of structural objects. Nevertheless it is important to stress that each of these accounts also comes with a different understanding of the reference of the mathematical vocabulary. Hence, we can say that the phenomena of ontological or definitional indeterminacy that motivate these structuralist ontologies directly translate into issues concerning the referential indeterminacy of mathematical terms. For instance, if the arithmetical theory $Q$ remains systematically indifferent with respect to the correct (set-theoretical) identification of natural number 2, it also leaves undecided the proper reference of numeral ' 2 '. ${ }^{39}$ Let us briefly look at two different accounts to deal with this kind of semantic indeterminedness based on two versions of eliminative structuralism.

The first version considered here is usually labelled universal structuralism. ${ }^{40}$ Universal structuralists hold that talk about the abstract structure of a mathematical theory is a way to paraphrase talk about all of its models. Thus, mathematicians use the term "abstract structure" as a way to generalize over all possible interpretations of a particular theory. This informal idea is usually made precise in terms of the following logical reconstruction of mathematical statements: take, for instance, the language of arithmetic $\mathcal{L}_{A}$ with signature $\{\mathrm{S},+, *, \leq\}$ in which our theory $\mathrm{Q}$ is formulated. As was shown above, every arithmetical sentence can be expressed in terms of the primitive vocabulary of $\mathcal{L}_{A}$, that is as a sentence of the form $\varphi[\mathrm{S},+, *, \leq]$. The central idea underlying universal structuralism is that the semantic content of such a statement is best captured by the following universally quantified conditional sentence:

$$
\forall f^{1} \forall f^{2} \forall f^{3} \forall r^{4}\left(\mathrm{Q}\left[f^{1}, f^{2}, f^{3}, r^{4}\right] \rightarrow \varphi\left[f^{1}, f^{2}, f^{3}, r^{4}\right]\right)
$$

\footnotetext{
${ }^{39}$ This semantic aspects related to structuralism, in particular the semantic indeterminacy of mathematical terms has been subject of recent discussions, in particular in [30], [49], and [41].

${ }^{40}$ See [45] and [40] for detailed discussions of this position.
} 
The consequent here is the formula resulting through the substitution of the primitive terms $\mathrm{S},+, *, \leq$ in sentence $\varphi$ by variables of the appropriate type. This syntactic reconstruction is motivated by the semantic assumption that ordinary mathematical statements do in fact express general claims about all possible interpretations of the background theory in question. Thus, a mathematical statement such as ' $2+2=4$ ' does not express a fact about a particular number system but about all possible systems of theory Q. ${ }^{41}$

Two points of commentary concerning this approach are in order here. First, given that universal structuralism does not posit a new mathematical ontology of structural objects, it is also sometimes labelled as "structuralism without structures" (see [48]). Nevertheless, the position does not come without ontological commitments. Notice that the formal reconstruction of mathematical statements has to be formulated in an interpreted language with quantifiers ranging over sets. It follows that universal structuralism in mathematics is not really universally eliminative (in the sense of applying to all mathematical domains) but presupposes a non-structural set theory as a background theory and thus a rich background ontology of sets. ${ }^{42}$ Universal structuralist are thus usually realists about sets, but nominalists about all other mathematical structures representable in the theory of sets.

Second, how should we think of the semantics of the primitive terms such as 'S', '+', ' * ', ' $\leq$ ' in light of the present account? Do these terms refer, and if so, to what? Presently, the most extensive discussion of this question is in [41]. According to what Pettigrew calls the antirealist or Aristotelian interpretation, such primitives as best understood as free variables (or as parameters) and not as constant expressions. More specifically, he argues that mathematical primitives act semantically similarly to the way variables do in the context of natural deduction, in particular under the standard rules of existential elimination and universal quantifier introduction. ${ }^{43}$

\footnotetext{
${ }^{41}$ In the present example, $\varphi^{*}$ can be viewed as a structuralist translation of statement $\varphi$ that is given in a "purified" (second-order) object language, i.e. a language with an empty signature. It can be viewed as an object-language translation of the underlying metatheoretical claim, namely that $\varphi$ is a logical consequence of theory Q. See[45] for further details.

${ }^{42}$ This set-theoretic ontology assumed here is usually specified by an axiomatic set theory, for instance ZFC.

${ }^{43}$ Compare Pettigrew on this analogy:

Thus, in a first-order system of natural deduction, free variables allow us to express generalities and to reason about them: in the case of universal introduction, we note that, if we can derive $\Phi(a)$ without making any assumptions about $a$, then we may conclude that, for any $a, \Phi(a)$; and, in the case of existential elimination, if we have that, for some $a, \Phi(a)$, and, whatever $a$ is, if $\Phi(a)$, then $B$ (where $B$ says nothing of $a$ ), then we can infer $B$. For this reason, it is not surprising that mathematical discourse contains a great many expressions that behave in its non-formal discourse exactly as free variables behave in formal systems of natural deduction.
} 
As mentioned above, one way to describe the semantic role of instantial terms in this context is the so-called quantificational account (see again [10] for a detailed discussion). This is the idea that the variables in question can be viewed as being tacitly bound by quantifiers. The very same understanding seems also viable in the context of mathematics. For instance, arithmetical primitives such as the '+'-operator can be viewed to function as a variable that ranges over all possible addition-functions in all possible number systems. As Pettigrew points out, we do not want the primitive variables to range over absolutely everything, i.e. over every admissible function in the set-theoretic universe, however. Instead, the mathematical terminology needs to be bound by certain theoretical constraints that can be specified relative to a given mathematical context. Specifically, the range of the same 'primitive' variables can be effectively relativized to different theoretical contexts in which they occur. ${ }^{44}$ In the translation scheme $\varphi^{*}$ presented above, this context relativity is represented formally by the antecedent of the conditional statement, i.e. by the relativization to the interpretation of the arithmetical terms to a particular theory, in the above case by $Q$.

Universal structuralism and the corresponding quantificational account of mathematical terms provide a natural explanation of modern structural mathematics as well as of the Benacerraf phenomena described above. Moreover, the account corresponds closely to the general "structuralist thesis" for scientific theories described above. In particular, the generality of theoretical knowledge is directly captured here in terms of the suggested reconstruction of a mathematical statement $\varphi$ by $\varphi^{*}$ : nothing is assumed here about the interpretation of the primitive terms but their logical category.

There exists a second version of eliminative structuralism in which this undeterminedness of mathematical terms is captured in a different way. This position has been labelled "relative structuralism" in [45] and shares many of the features of universal structuralism. In particular, it also assumes a non-structural set theory in which models of mathematical theories can be constructed. Moreover, it is also clearly deflationist in the sense that mathematical structures are not treated as bona fide abstract objects. In contrast to universal

After all, mathematics deals in generalities. [41, p.313]

\footnotetext{
${ }^{44}$ Pettigrew therefore describes primitive terms as "contextually dedicated free variables". The range of the variables $0,1,+, \times$ depends on the specific context in which they are introduced. Contexts can be theoretical constraints such as: (1) ' $\mathbb{R}, 0,1,+, \times$, and $<$ satisfy the axioms for a complete ordered field'; ' $\mathbb{C}, 0,1,+, \times$, and $<$ satisfy the axioms for a complex field'; ' $R, 0,1,+$, and $\times$ satisfy the ring axioms'. [41, pp.316-317]
} 
structuralism, talk about structures is not understood as a way to generalize over models, however. Instead, structures are understood here as arbitrary representatives of the model class of a theory.

Moreover, unlike in universal structuralism, the mathematical primitives of a theory are not considered as variables, but as constant expressions with determinate referents in a given model. What distinguishes this structuralist position from more traditional realist accounts is a certain "relativity of reference" typical for such terms. Thus, the primitive terms of a mathematical theory are treated as constant expressions that refer to specific objects (i.e. individuals, relations, or functions) in one of its models. Their reference, however, is specified relative to an arbitrary choice of such a model. Thus, one can pick out any model in question and fix the the reference of the theory's terms relative to this choice. Consider, for instance, the language or second-order Peano arithmetic with signature ' $s$ ' (for 'successor') and ' 1 '. How are these terms interpreted according to the present account? Reck \& Price give the following explanation:

A relativist structuralist offers the following response: We simply pick one particular model $M$ of $P A_{2}$, of a domain $S$, a distinguished element $e$ in $S$, and a successor function $f$ on $S$ (here $M$ can be some model that is particularly convenient for the purposes at hand, but it doesn't have to be); and we stipulate that ' 1 ' refers to $e$, that ' $s$ ' refers to $f$, and that the range of the quantifiers is $S$. At the same time, we note that we could also have picked any other model $M^{\prime}$ of $P A_{2}$. (...) Still, having made it we keep our initial stipulation fixed until further notice. [45, p.349]

Two aspects of this understanding of the semantics of mathematical terms should be emphasized here. The first is the relativity of the interpretation function, i.e. the specification of reference relative to a chosen model and second. The second is arbitrariness of this model selection. The reference of the primitive terms can thus be determined relative to any possible model, that is, one can pick out any object from the model class in question and fix their reference relative to this choice. In the above example, any number system satisfying $P A_{2}$ can in principle be considered for the specification of the reference of terms ' $s$ ' and ' 1 '.

Why should this account be viewed as a kind of structuralism concerning mathematical theories? The central structuralist insight here is again a semantic point, namely that the mathematical reference is stipulated arbitrarily with respect to a given model class. 
Compare again Reck \& Price on this point:

That is to say, relativist structuralism works with a notion of reference (modeled on the notion of interpretation in model theory) that is relative to such a choice - thus its name. On the basis of such reference it is also determined what is meant by "the natural numbers"; namely the particular model $M$ of $P A_{2}$ that has been chosen initially. Of course this choice is largely arbitrary, since we could have picked any other model of $P A_{2}$ instead. But that does not matter. All that matters, from this point of view, is that we are consistent about our choice. [45, p.349]

It is essentially the arbitrariness of the (model-theoretic) interpretation mentioned here that makes the present position into a structuralist one. In a slogan, one could say that relative structuralism is the combination of a set-theoretic realism (i.e. a realism about sets) with a theory of arbitrary reference for mathematical terms. ${ }^{45}$

Relative structuralism corresponds closely to the structuralist thesis sketched above. The generality of theoretical knowledge typical for the latter is not captured here in terms of the generalization over possible interpretations however, but in terms of an arbitrary choice of such an interpretation. It should also be clear that this 'relativist' account of structuralism squares particularly well with Carnap's epsilon-reconstruction of theories. More specifically, the explicit definition of theoretical terms presented in Section 3 gives an explication of the semantic behaviour of mathematical primitives that is in direct accord with this philosophical view. Notice in particular that the epsilon-term definition captures the two central desiderata of relative structuralism described above, namely that (i) the reference of primitive terms is specified relative to a given choice of a model (of the theory in question) and (ii) that the choice of this model is arbitrary. To illustrate this, consider again the reconstruction of the theory of monoids presented in Section 3. The epsilondefinition of the tuple of primitive terms $t=\langle G, \circ, e\rangle$ was explicitly defined here by:

$$
t={ }_{d f} \epsilon_{z}(\exists X \exists f \exists v(z=\langle X, f, v\rangle \wedge \operatorname{Mon}(X, f, v))
$$

The theoretical context relative to which the interpretation of tuple $t$ (and thus, indirectly, of each primitive term) is determined is captured by the complex formula $\operatorname{Mon}(X, f, v)$ that

\footnotetext{
${ }^{45}$ The fact that mathematical primitive terms are taken to refer arbitrarily is not so untypical of mathematical discourse. As pointed out in [36] and more recently, in [10], the use of arbitrary terms is quite common in mathematical reasoning, for instance in stipulative sentences occurring in mathematical proofs. What the present account shows is that this idea of arbitrary reference can be generalized to hold not only for uses of instantial terms in mathematical reasoning, but for mathematical discourse in general.
} 
expresses the conjunction of the monoid axioms. Possible assignments to the elements in tuple $t$ are specified relative to the model class defined by this formula. ${ }^{46}$

The second main desideratum of relative structuralism, namely that the reference of the mathematical terms is specified relative to an arbitrary model selection, is directly captured by the use of an epsilon-operator in the above definition. Thus, the reference of terms ' $G$ ', ' $\circ$ ', and ' $e$ ' is specified by their defining epsilon-terms and a modern choicesemantical interpretation of those. In the specific example, the epsilon operator picks out one arbitrary representative from the class of monoids. The local interpretation of the primitive terms is then specified relative to this choice.

Given this formal framework for relative structuralism, we can offer a general translation scheme for mathematical statements similar to the translation scheme usually associated with universal structuralism. We say that a mathematical statement of the form $\varphi(t)$ - where $t$ is again the tuple of the mathematical primitives of a theory $T$ - can be reconstructed as:

$$
\varphi\left(\epsilon_{x}(T(x))\right)
$$

where tuple $t$ is simply replaced by the epsilon-definiens constructed in the above way. ${ }^{47}$ Carnap's epsilon-reconstruction thus gives us a way to make precise this intuitive version of mathematical structuralism and the semantics of mathematical discourse that comes with it.

\section{Conclusion}

In this paper, we presented a modernized account of the logic and semantics underlying the epsilon-reconstruction of theories and applied it to the context of pure mathematics. Our guiding philosophical motivation was to evaluate a recent observation (made in work by Friedman, Demopoulos, and others) that Carnap's mature logic of science is compatible

\footnotetext{
${ }^{46}$ We can thus say that this pure formula (or its Ramsification) functions similarly here to the way Pettigrew speaks of theoretical contexts and "contextually dedicated variables" in his account of universal structuralism. Unlike in Pettigrew's account, however, theoretical constraints are not specified externally but internally, i.e. in the definiens of the relevant terms.

${ }^{47}$ Notice that in this structuralist reconstruction of mathematical statements, much depends on the choice of the theoretical context $\mathrm{T}$ here. The semantic evaluation of a statement can vary significantly relative to different background theories. Say, for instance, that for a given statement expressed in a general algebraic terminology, we can specify as our background theory either the theory of monoids or the theory of abelian groups. The model selection given by the epsilon-operator can lead to different truth values of the statement in question.
} 
with scientific structuralism. To address this, we gave a logical analysis of the indefinite character of the epsilon operator as well as of the specific type of reference usually associated with epsilon terms. As was argued, this type of arbitrary reference characteristic of Carnap's understanding of theoretical terms connects his account with scientific structuralism. More specifically, it was shown that the epsilon-term definition of theoretical vocabulary - in our case of the primitive terms of theories of pure mathematics - also suggests an explication of the informal semantics such terms have according to a particular structuralist conception of mathematics.

Given this, two lines of possible future research on the epsilon reconstruction and its philosophical interpretation should be mentioned briefly here. First, what has not been addressed in detail here is how our analysis of Carnap's method applies also to nonmathematical branches of sciences and to scientific structuralism more generally. These connections are in our view less obvious than those to the philosophy of mathematics, mainly for two reasons: first, it is less clear than in the case of mathematics how to specify formally what a scientific theory is and also how to conceive of the semantics of its theoretical terminology. Moreover, unlike in the case of pure mathematics, it is also less established what a structuralist conception of theories in the context of the natural sciences really amounts to. For instance, the central idea underlying epistemic structural realism, namely that scientific theories describe only structural properties of the world, has been cashed out formally in different ways (see, e.g., [8], [18], and [21]). Moreover, there exists a broad spectrum of diverging views in ontic structural realism on how the specify the metaphysical notion of the structure of the world. ${ }^{48}$ A study of possible points of contact between Carnap's approach and these structuralist positions in the philosophy of science will thus depend on a closer analysis of how the epsilon-reconstruction relates to different versions of structural realism.

Second, it might be interesting to see how Carnap's epsilon-reconstruction can be applied to other topics under discussion in general philosophy of science and scientific structuralism in particular. This concerns, in particular, issues relevant in the philosophical discussion of non-revolutionary theory change. In the present paper, we have assumed a 'static' account of theories, that is a focus on individual theories, considered in isolation. In contrast, recent work on scientific structuralism is primarily concerned with dynamic processes, for instance the supersession of one theory by another. Applying Carnap's ac-

\footnotetext{
${ }^{48}$ Consider, e.g., [5] and [1] for excellent overviews of the current state of debate.
} 
count to such a dynamical framework might allow us to make logically precise several critical notions in the philosophical discussion of scientific theory change. Two topics seem particularly noteworthy here. The first concerns the referential continuity of theoretical terms in theory change. Carnap's epsilon-term definition - and in particular its modern choice semantical specification - might allow us to formulate a weak but precise criterion for referential continuity. ${ }^{49}$ The second topic concerns the notion of structural continuity in theory change that plays a central role in the debates on epistemic structural realism since Worrall's [51]. The underlying idea here is that there is a continuity between theories and their successor theories on a purely structural level, that is, continuity in terms of shared structural content. Carnap's epsilon account of theories might be put to use here again in order to give an alternative precise characterization of this notion. Both topics will be dealt with in future work.

\section{References}

[1] P. M. Ainsworth. What is ontic structural realism? Studies in History and Philosophy of Science Part B, 41(1):50-57, 2010.

[2] H. Andreas. A modal view of the semantics of theoretical sentences. Synthese, 174:367$383,2010$.

[3] H. Andreas. Theoretical terms in science. The Stanford Encyclopedia of Philosophy, http://plato.stanford.edu/archives/sum2013/entries/theoretical-terms-science/, 2013.

[4] G. Asser. Theorie der logischen Auswahlfunktionen. Zeitschrift für mathematische Logik und Grundlagen der Mathematik, 3:30-68, 1957.

[5] J. R. Becker Arenhart and O. Bueno. Structural realism and the nature of structure. European Journal for Philosophy of Science, 5(1):111-139, 2015.

[6] J. L. Bell. Hilbert's epsilon operator in intuitionistic type theories. Mathematical Logic Quarterly, 39:323-337, 1993.

[7] P. Benacerraf. What numbers could not be. Philosophical Review, 74:47-73, 1965.

\footnotetext{
${ }^{49}$ Compare, for instance, [44] on this point.
} 
[8] A. Bokulich and P. Bokulich, editors. Scientific Structuralism. Boston Studies in the Philosophy and History of Science, Vol. 281. Dordrecht: Springer, 2011.

[9] K. Brading and E. Landry. Scientific structuralism: presentation and representation. Philosophy of Science, 73:571-581, 2006.

[10] W. Breckenridge and O. Magidor. Arbitrary reference. Philosophical Studies, 158(377400), 2012.

[11] J. Burgess. Putting structuralism in its place. (unpublished manuscript).

[12] J. Busch. What structures could not be. International Studies in the Philosophy of Science, 17(3):211-225, 2003.

[13] R. Carnap. Foundations of Logic and Mathematics. International encyclopedia of unified science, vol.I. Chicago: University of Chicago Press, 1939.

[14] R. Carnap. The methodological character of theoretical concepts. In H. Feigel and M. Scriven, editors, Minnesota studies in the philosophy of science, vol. I: The foundations of science and the concepts of psychology and psychoanalysis, volume 1, pages 38-76. Minneapolis: University of Minnesota, 1956.

[15] R. Carnap. Beobachtungssprache und theoretische Sprache. Dialectica, 12(236-248), 1958.

[16] R. Carnap. On the use of Hilbert's $\epsilon$-operator in scientific theories. In Y. Bar-Hillel et al., editor, Essays on the foundations of mathematics, pages 156-164. Jerusalem: The Magnus Press, 1961.

[17] R. Carnap. Replies and systematic expositions. In P. A. Schilpp, editor, The philosophy of Rudolf Carnap, pages 859-1013. LaSalle: Open Court, 1963.

[18] A. Cei and S. French. Looking for structure in all the wrong places: Ramsey sentences, multiple realisability, and structure. Studies in History and Philosophy of Science, 37:633-655, 2006.

[19] J. C. Cole. Mathematical structuralism today. Philosophy Compass, 5(8):689-699, 2010. 
[20] W. Demopoulos. On the rational reconstruction of our theoretical knowledge. British Journal for the Philosophy of Science, 54(371-403), 2003.

[21] W. Demopoulos. Logicism and its Philosophical Legacy. Cambridge: Cambridge University Press, 2013.

[22] K. Fine. Reasoning With Arbitrary Objects. Aristotelian Society Series. Blackwell, 1985.

[23] S. French. The interdependence of structure, objects and dependence. Synthese, 175(S1):89-109, 2010.

[24] M. Friedman. Wissenschaftslogik: The role of logic in the philosophy of science. Synthese, 164:385-400, 2008.

[25] M. Friedman. Carnap on theoretical terms: structuralism without metaphysics. Synthese, 180:249-263, 2011.

[26] N. Gratzl. Ambiguous descriptions. (unpublished manuscript).

[27] P. Hajék and H. Pudlák. Metamathematics of First-Order Arithmetic, volume 2. Berlin: Springer, 1998.

[28] C. G. Hempel. The theoretician's dilemma: A study in the logic of theory construction. In M. Scriven H. Feigl and G. Maxwell, editors, Minnesota studies in the philosophy of science, vol. II: Concepts, theories and the mind-body problem, pages 37-98. Minneapolis: University of Minnesota Press, 1958.

[29] C. G. Hempel. The meaning of theoretical terms: A critique of the standard empiricist construal. In A. Joja P. Suppe, L. Henkin and G. C. Moisil, editors, Logic, Methodology and Philosophy of Science IV, pages 367-378. Amsterdam, 1973.

[30] K. Hodesdon. Mathematical representation: playing a role. Philosophical Studies, DOI 10.1007/s11098-013-0160-4, forthcoming.

[31] J. King. Instantial terms, anaphora and arbitrary objects. Philosophical Studies, 61:239-265, 1991.

[32] J. Ladyman. What is structural realism? Studies in History and Philosophy of Science, 29(3):409-424, 1998. 
[33] A. C. Leisenring. Mathematical Logic and Hilbert's Epsilon-Symbol. London: Macdonald, 1969.

[34] D. Lewis. How to define theoretical terms. The Journal of Philosophy, 67(13):427-446, 1970.

[35] Ø. Linnebo. Structuralism and the notion of dependence. Philosophical Quarterly, 58:59-79, 2008.

[36] E. Martino. Arbitrary reference in mathematical reasoning. Topoi, 20:65-77, 2001.

[37] G. Maxwell. Structural realism and the meaning of theoretical terms. In S. Winokur and M. Radner, editors, Minnesota Studies in the Philosophy of Science, vol. IV: Analyses of Theories and Methods of Physics and Psychology, pages 181-192. Minneapolis: University of Minnesota Press, 1970.

[38] G. M. Mints and D. Sarenac. Completeness of an indexed epsilon-calculus. Archive for Mathematical Logic, 42:617-625, 2003.

[39] G. Moser and R. Zach. The epsilon calculus and Herbrand complexity. Studia Logica, 82(1):133-155, 2006.

[40] C. Parsons. The structuralist view of mathematical objects. Synthese, 84(3):303-346, 1990.

[41] R. Pettigrew. Platonism and aristotelianism in mathematics. Philosophia Mathematica, 16(3):310-332, 2008.

[42] S. Psillos. Scientific Realism. London: Routledge, 1999.

[43] S. Psillos. Carnap, the Ramsey-sentence and realistic empiricism. Erkenntnis, 52(253279), 2000.

[44] S. Psillos. Rudolf Carnap's 'Theoretical concepts in science'. Studies in History and Philosophy of Science, 31:151-172, 2000.

[45] E. Reck and M. Price. Structures and structuralism in contemporary philosophy of mathematics. Synthese, 125(3):341-383, 2000.

[46] M. Resnik. Mathematics as a Science of Patterns. Oxford: Claredon Press, 1997. 
[47] B. Russell. Introduction to Mathematical Philosophy. London: George Allen and Unwin; New York: The Macmillan Company, 1919.

[48] S. Shapiro. Philosophy of Mathematics: Structure and Ontology. New York: Oxford University Press, 1997.

[49] S. Shapiro. Identity, indiscernibility, and ante rem structuralism: the tale of i and -i. Philosophia Mathematica, 3(16):285-309, 2008.

[50] K. von Heusinger. The reference of indefinites. In K. von Heusinger and U. Egli, editors, Reference and Anaphoric Relations, pages 265-284. Dordrecht: Kluwer, 2000.

[51] J. Worrall. Structural realism: the best of both worlds? Dialectica, 43:99-124, 1989.

[52] R. Zach. The practice of finitism. Epsilon calculus and consistency proofs in Hilbert's Program. Synthese, 137:211-259, 2003.

[53] R. Zach. Lectures on the epsilon calculus. Lecture notes, 2009. 